\title{
COMMUNICATIONS
}

\section{THREE-COLOUR DEMONSTRATION OF ARTERIAL VENOUS AND AQUEOUS OUTFLOW ROUTES*†}

\author{
BY \\ R. K. MACDONALD AND T. M. SIBAY \\ From the Department of Ophthalmology, Faculty of Medicine, University of Toronto
}

SiNCE Ashton (1951, 1952), using the Neoprene cast technique, demonstrated the relationships between the aqueous veins of Ascher (1942), the canal of Schlemm, and the closely related arterial network (Ashton and Smith, 1953), the literature has been richly provided with findings and speculation on the likely role of each vessel in the control of intra-ocular pressure. Schwalbe (1870) and Leber (1873) had shown evidence of aqueous "lymph" channels by injecting dyes into the anterior chamber, but since that time only an impression of the vascular pattern had been built up, largely from serial sections.

In Ashton's original work, Neoprene, injected into the canal of Schlemm, made its way readily into the aqueous outflow channels. The tissues were then dissolved by digests leaving intact a Neoprene cast of the canal of Schlemm and its outflow channels. In some specimens the arterial system was also injected with a different colour of Neoprene so that the close relationships between the arterial and aqueous channels could be observed.

While Ashton's work provides a most valuable appreciation of the aqueous outflow pathways and their arterial relationships in the human eye, his technique is not applicable to studies on some experimental animals, notably the rabbit. The albino rabbit is a convenient laboratory animal in which to study the relationships between the arterio-venous and the aqueous outflow systems.

The rabbit eye like the human eye has numerous outflow channels which have multiple venous connexions and closely related arteries. It does not, however, possess a canal of Schlemm into which to inject a substance of large molecular size, such as Neoprene. A different, and easily repeatable, technique was therefore sought, in which some dye injected directly into the anterior chamber would make its appearance in the outflow channels by diffusion through the meshwork at the angle.

Numerous methods and substances requiring passage from the anterior chamber through the angle meshwork are on record to demonstrate the channels of aqueous outflow.

\footnotetext{
* The study was supported by a Public Health Grant.

+ Received for publication February 9, 1962.

46
} 
In vivo, slit-lamp observations have been made on the entry of fluorescein into the aqueous veins after injection into the anterior chamber (Goldmann 1946). Radiographically, sodium diatrizoate, injected into the anterior chamber of the rabbit eye, has been shown leaving via two different aqueous channels (Cohan, 1958): one directly over the angle meshwork, and the other, slightly more posteriorly, over the ciliary body. At the latter site there is thought to be communication with the anterior ciliary venous plexus. In the cat the same substance injected into the anterior ciliary vein has been used to demonstrate the scleral and uveal plexus (Cohan, 1960).

In vitro, such diffusible solutions as silver or starch injected into the anterior chamber may, with a good degree of success, be identified and fixed in the aqueous outflow channels. Similarly, suspensions of finely-divided particles, such as India ink, colloidal silver, colloidal iron (Cibis and Yamashika, 1959), and particles as large even as red blood cells (citrated), have been used to demonstrate the aqueous outflow channels.

When attempts are made, however, to demonstrate three systems: the aqueous outflow, the arterial, and the venous system, each in a different colour in the same specimen, several difficulties present themselves, apart from the obvious one of inadvertently displacing one colour by another. Many substances are found unsuited to perfusion from the anterior chamber because of the high resistance offered to the perfusate by the filtration meshwork. Interestingly enough, a substance blocked by the filtration meshwork is, in most instances, if perfused arterially, blocked at the precapillary level.

Particle size is not the only criterion for an ideal perfusate. Stable colloids, such as India ink, although passing easily through the filtration meshwork into the scleral outflow channels, tend to be expressed from the scleral channels during subsequent handling of the tissues, disguising the location of the outflow pathway. Even mixing gelatin with the ink and cooling after perfusion do little to overcome this problem.

Less stable colloids, although exhibiting excellent staining characteristics, have a marked tendency to precipitate in the filtration meshwork, or if perfused arterially, in the precapillary arterioles. Dyes of small molecular size, such as Evan's blue, although passing easily through the filtration meshwork (Greaves and Perkins, 1952), cannot be fixed adequately and spread too widely through the tissues.

In consideration of these complicating factors, a technique is here described in which three brightly contrasting coloured substances stain the arteries, the veins, and the aqueous outflow channels, each in its own colour. Where the venous and aqueous flow merge, a fourth easily distinguishable colour is produced. The specimen is cleared and studied stereoscopically.

\section{Method}

An albino rabbit, approximately 6 months old, is first heparinized intravenously $(1,000$ units per $\mathrm{kg}$. body weight). After 5 minutes, the rabbit is given a lethal 
intravenous injection of Nembutal. Through an abdominal opening the inferior vena cava is identified and incised to allow for subsequent perfusion outflow. With the rabbit in the supine position the carotid artery is identified on each side of the trachea, deep to the strap muscles. Dissection is done with blunt instruments to avoid severing vessels, arterial or venous. Care being taken to avoid air entry, each artery is cannulized at a level inferior to the larynx by a blunt, slightly curved, 19-gauge needle attached to a $20 \mathrm{ml}$. syringe filled with saline. At the rate of approximately $1 \mathrm{ml}$. per sec., $20 \mathrm{ml}$. saline are then injected into each artery.

If saline perfusion is adequate, the limbal vessels, as well as the pink choroidal and iridial reflex in the ipsilateral eye, gradually clear. Occasionally an additional $10 \mathrm{ml}$. saline must be injected to complete clearing. Further perfusion is usually undesirable because of resultant tissue swelling. Should no clearing occur, the needle must be re-inserted in the artery more proximally, to allow for anomalous circulation. Often the vessels of the pinna remain uncleared of blood, but this was found to be unimportant to the end-results.

After saline perfusion, formo-gelatin solution is injected in the same manner, 20 ml. to each artery.* During formo-gelatin perfusion small muscle twitchings may be noted throughout the body.

To prevent a rise in intra-ocular pressure, a 30-gauge needle is inserted tangentially through the cornea into the aqueous chamber and left in situ.

Following formo-gelatin, Monolite red mixture is perfused, $10 \mathrm{ml}$. to each side. $\S$ As the Monolite red mixture is being injected, the tissues, chiefly on the ipsilateral side, turn a healthy pink, and the limbal vessels, ciliary processes, iris, and choroidal vessels turn a deep scarlet.

Perfusion of Monolite red mixture is immediately followed by injection, $15 \mathrm{ml}$. to each side, of Viacutan-formalin mixture. $\dagger$ As the Viacutan-formalin mixture is being injected, the scarlet limbal iridial vessels pale somewhat. This is assurance of good perfusion.

Viacutan perfusion is followed by Monolite red mixture, again $10 \mathrm{ml}$. to each side. The animal is left undisturbed for approximately $10 \mathrm{~min}$. At the end of this time, the limbal vessels remain faint pink even if they are purposely emptied by local massage. Formo-gelatin, $5 \mathrm{ml}$. to each side, is then injected arterially.

Arterial injection is now complete and is immediately followed by perfusion through the filtration meshwork with Congo red mixture. $\ddagger$ A 2-ml. syringe containing Congo red mixture is fitted to the corneal needle. Some mixture is injected and withdrawn two or three times to ensure even mixing throughout the

* Formo-gelatin is prepared by first dissolving $0.35 \mathrm{~g}$. gelatin in $100 \mathrm{ml}$. warmed distilled water. With this solution at room temperature, $2.5 \mathrm{ml}$. stock formalin solution are added and mixed.

$\S$ Monolite red mixture is made up of ingredients in the following order:

$\begin{array}{lr}\text { Monolite red 4RHVS paste } & 10 \mathrm{ml} \text {. } \\ 10 \text { per cent. Dispersol OG (in distilled water) } & 5 \mathrm{ml} \text {. } \\ \text { Gelatin sol. } 0.35 \text { per cent. (in distilled water) } & 50 \mathrm{ml} \text {. }\end{array}$

Monolite red 4RHVS paste and 100 per cent. Dispersol OG is available commercially from Imperial Chemical Industries Ltd., Blackley, Manchester 9, England.

† To $200 \mathrm{ml}$. distilled water $6 \mathrm{~g}$. Viacutan powder are added and mixed. The fluid is filtered and stored in a dark bottle. Just before perfusion $5 \mathrm{ml}$. stock formalin solution are added and stirred. Viacutan is commercially available from Ward Blenkinsop \& Co., 6 Henrietta Place, London, W.1., England.

$\ddagger$ In the preparation of Congo red mixture $0.35 \mathrm{~g}$. gelatin is dissolved in $35 \mathrm{ml}$. warmed distilled water. Into another $35 \mathrm{ml}$. distilled water is dissolved $0.25 \mathrm{~g}$. Congo red. These two solutions are mixed at room temperature and then added to $35 \mathrm{ml}$. absolute ethyl alcohol. 
anterior chamber. Then, at a pressure sufficient to cause corneal clouding, perfusion is carried out for approximately 1 minute. As soon as an orange-red hue is imparted to the formerly pale scarlet limbal vessels, an assistant instills acid fixative§ on the eye.

After perfusion, the contents of the anterior chamber are quickly withdrawn. With the needle still in the cornea, the syringe is disconnected and replaced by one containing acid fixative. The chamber is then irrigated at low pressure. High pressure with acid fixative causes undesirable swelling of the cornea and sclera. On contact with acid the entire anterior chamber and the red limbal vessels turn deep blue in colour.

The eye is then enucleated and excised around the equator. The lens and vitreous are dislodged and the anterior segment is immersed in acid fixative for approximately 30 seconds. The specimen is then cut sagittally with scissors from the free edge of the sclera inferiorly, at 6 o'clock, to the superior edge of the cornea near the limbus, at 12 o'clock. The specimen can now be spread out as a flat, arch-shaped preparation if a few suitable short scissor cuts are made in the free edges. The limbus should be left intact. Similarly the iris is cut radially in four or five places, from the pupillary margin to its base. The cut segments of iris are drawn posteriorly with toothed forceps, partially tearing the iris root and part of the ciliary body from their scleral attachments. A blue-staining band of sclera at the filtration angle is thereby denuded (Fig. 1a, $b$ ).

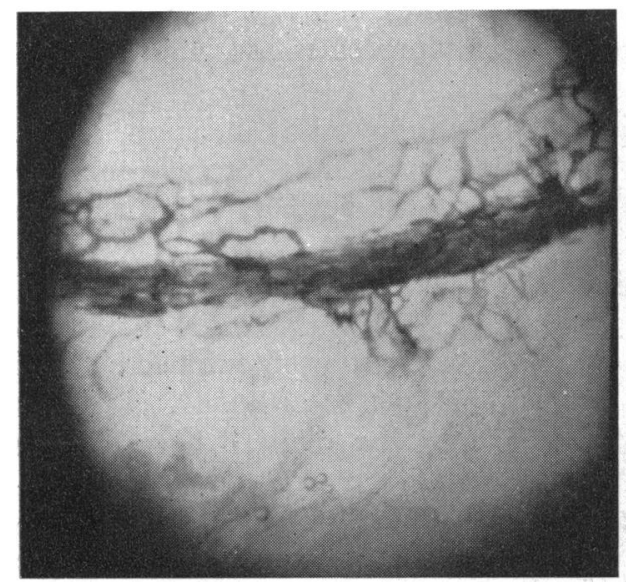

(a)

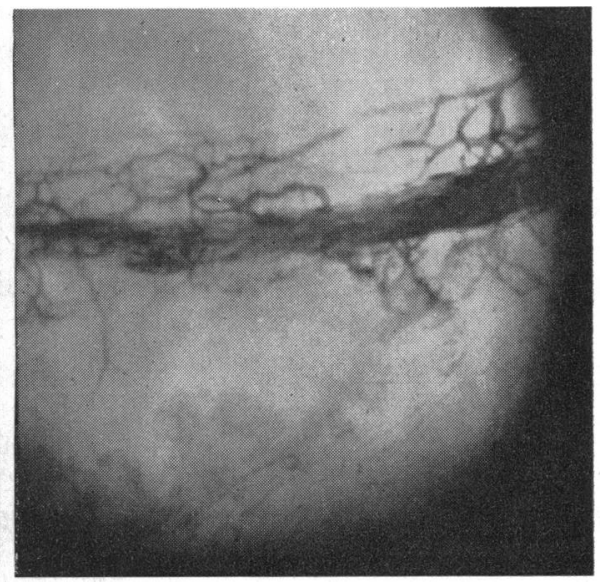

(b)

FIG. 1 ( $a$ and $b$ ).-Stereophotomicrograph, showing relationship of anterior and posterior aqueous outflow channels to filtration meshwork. $\times 12$.

The latter blue stain is gently scraped away with a curved Bard-Parker knife. This uncovers a series of little blue dots, the inner openings of the aqueous channels. Blue staining Descemet's endothelium is similarly scraped away.

$\$$ Acid fixative is prepared as follows:

$\begin{array}{ll}20 \text { per cent. hydrochloric acid } & 200 \mathrm{ml} . \\ \text { Absolute ethyl alcohol } & 200 \mathrm{ml} . \\ \text { Sodium chloride } & 6.8 \mathrm{~g} .\end{array}$


On the external surface of the specimen, the conjunctiva and subconjunctival tissues are removed to avoid interference with visual examination of the deeper structures.

The specimen is then dipped in glycerol and pressed between two glass slides with the iris segments still folded posteriorly. After one minute between the slides the somewhat hardened flat specimen is removed and immersed in glycerol for 10 minutes for complete clearing. During the clearing period the specimen is subjected to bright light to develop the silver stain. Developing is greatly enhanced by direct exposure to ultraviolet light for 1 or 2 minutes, during which time the entire arterial network becomes etched in brown. This becomes desirably yellowish if warmed by ultraviolet light.

The preparation is now ready for study, the entire procedure having taken less than one hour. Stereoscopic observations are generally made by stereomicroscopy at $\times 40 \times 80$ magnification with transmitted and reflected light.

The specimen must be stored in glycerol at a temperature below $0^{\circ} \mathrm{C}$.

\section{General Observations}

With good perfusion technique, clearing of blood from the limbal plexus occurs fairly rapidly in the ipsilateral eye and more slowly in the contralateral eye. If the major veins of the neck are severed during dissection, insufficient perfusate reaches the orbital area. Too much saline and too great pressure (during perfusion) cause swelling of the tissues of the orbit. Gelatin mixed with some of the perfusates greatly inhibits tissue swelling.

Monolite red paste is a colloid which is precipitated by acids and by some tissue fluids. It is protected against the latter by Dispersol OG. If the protected colloid is injected into the anterior chamber, it can be forced through the filtration meshwork into the aqueous outflow channels.

Viacutan, even after it is filtered, is pale brown and slightly turbid. Because it is light-sensitive it must be freshly prepared and shielded from direct illumination. If the Viacutan is injected without gelatin, even at low pressures, marked muscle twitchings, mucosal gland secretion, and swelling of the vitreous with marked increase in intra-ocular pressure takes place. Viacutan also diffuses into the anterior chamber causing permanent blockage of the filtration meshwork. These undesirable effects may be prevented by mixing the Viacutan with gelatin solution.

Congo red solution is clear red and when injected passes easily through the filtration meshwork of the rabbit's eye into the anterior chamber at pressures as low as $15 \mathrm{~mm}$. Hg. Perfusion is even freer in the human and monkey eye, although the outflow facility for aqueous humour in these species is similar to that of the rabbit. Perfusion is not impaired by the presence of alcohol. Diffusion of Congo red through the capillary walls into the tissues is prevented by precipitating the dye with hydrochloric acid. When Congo red is mixed with 10 per cent. hydrochloric acid, a completely reversible reaction takes place, resulting in the formation of a deep blue flocculent precipitate. A more suitable fine blue suspension is formed if gelatin is present in the Congo red solution. Hydrochloric acid instilled on the eye penetrates the 
ocular coat in one minute, causing the Congo red in Descemet's membrane to turn blue.

At acid $\mathrm{pH}$ the conjunctiva swells slightly and loses transparency. This tissue is either excised or is later cleared in glycerol. Swelling of the specimen as well as loss of acidity is prevented later by storing at $0^{\circ} \mathrm{C}$.

\section{Microscopic Observations}

The three colours of the vascular systems in the relatively transparent preparation can readily be observed: the channels available to aqueous outflow staining blue (Fig. 2a,b), the arterial system brown (Fig. 3), and the venous and capillary network red (Fig. 3). Where the aqueous and venous channels merge a distinct purple colour is produced (Fig. 3). Occasionally, at the site of the merging, red and blue layering or stratification, similar to that observed clinically in the aqueous veins of Ascher, is seen (Fig. 4).

The arterial network appears brown in transmitted light and golden yellow in purely reflected light with a dark background. Viacutan stains the intercellular cement substance and the cell membrane (Hausler and Sibay, 1960). and produces cross striations in the larger arteriolar walls (Fig. 5).

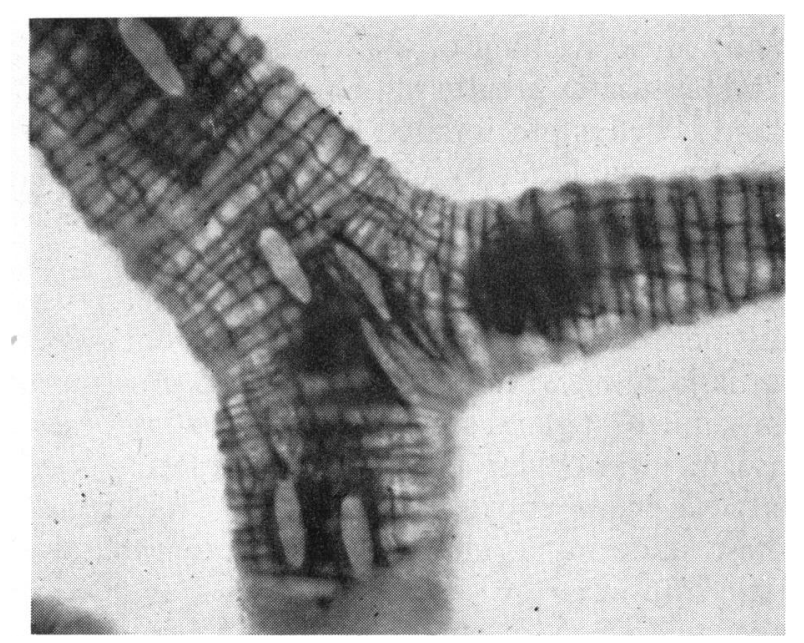

Fig. 5.-Human retinal arteriole stained with Viacutan showing cross-striations. $\times 750$.

Fig. 2 ( $a$ and $b$ ).-Stereophotomicrograph of limbus, showing aqueous outflow channels blue, limbal arcade red, and arterial system brown. $\times 16$.

FIG. 3.-Limbal area, showing mixing of aqueous (blue) and venous (red) currents. The arterial system is stained brown. $\times 20$.

FIg. 4.- Site of insertion of superior rectus muscle, showing stratification and mixing of aqueous (blue) and venous (red) currents. $\times 16$.

FIG. $12(a$ and $b$ ).-Stereophotomicrograph of ciliary processes, showing vascular communications with choroid. Note outer long ciliary arterial (brown) branches. The angle meshwork stains deep blue. $\times 16$. 

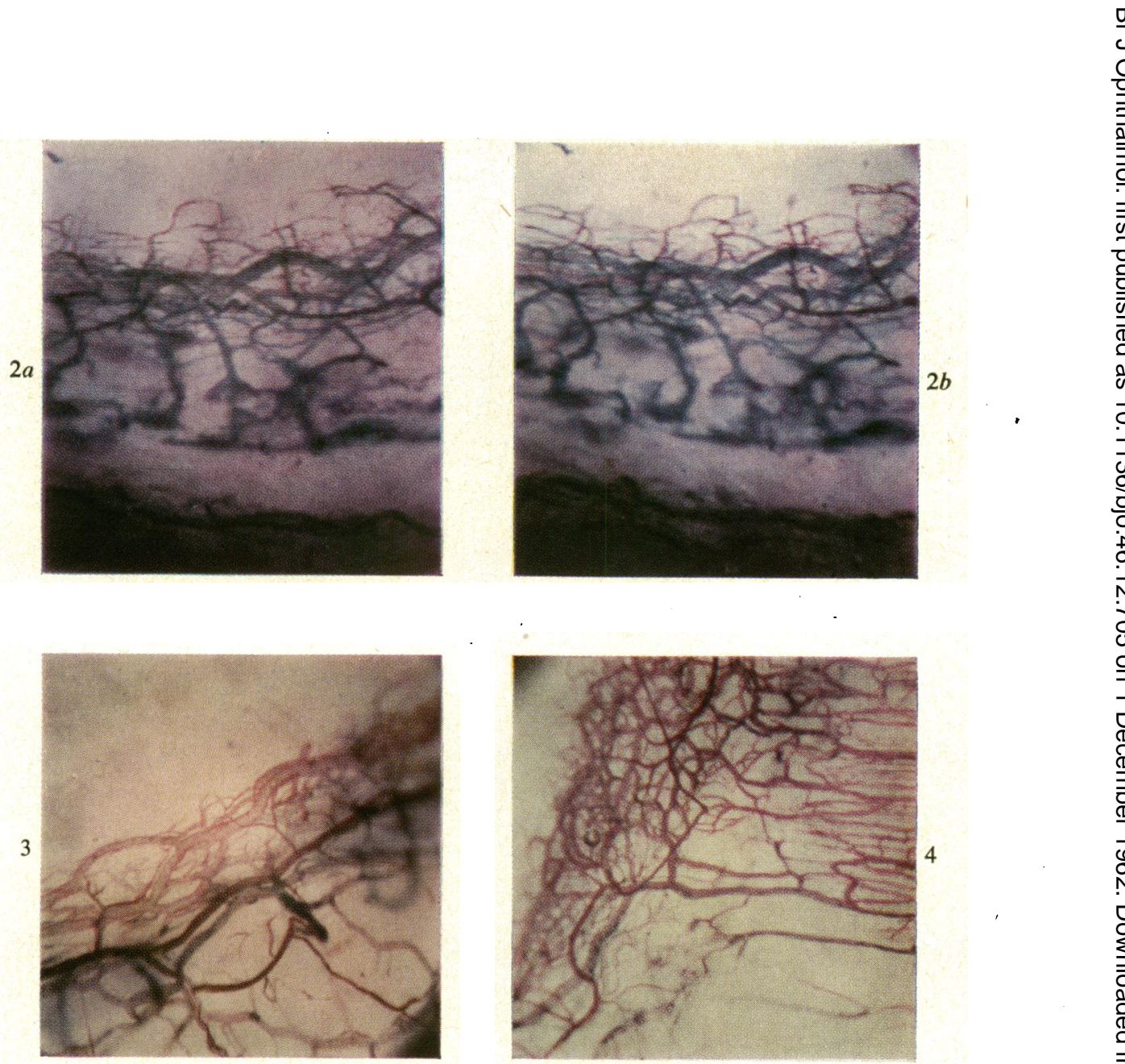

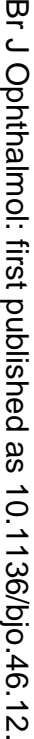

ชั

ㅇ

$\overrightarrow{0}$

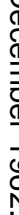
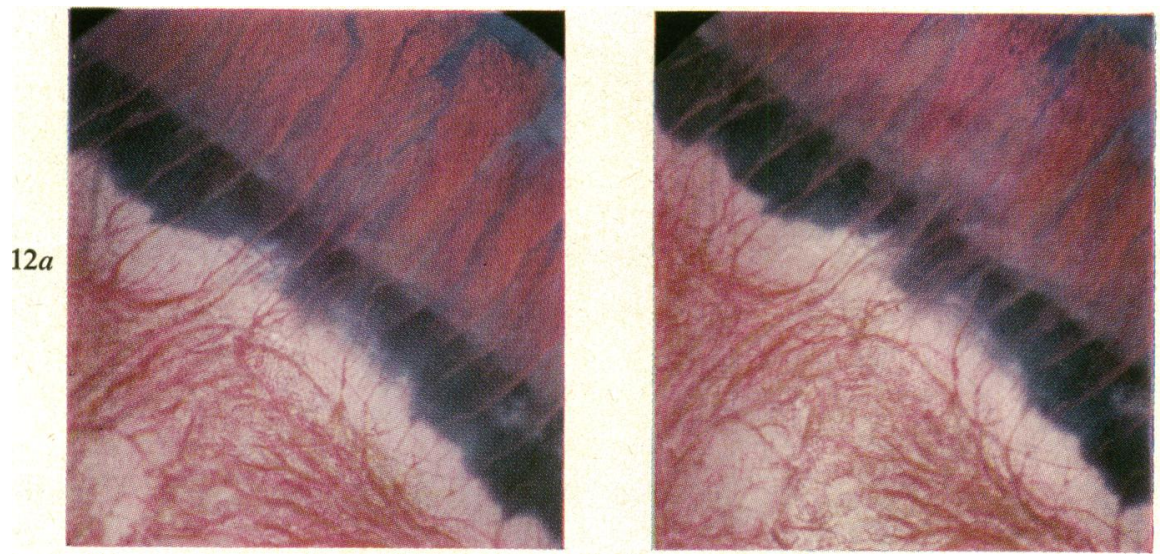

$12 b$

To face page 710]

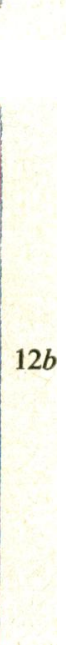


The arterial system is stained by Viacutan up to the precapillary arteriolar level (Fig. 6).

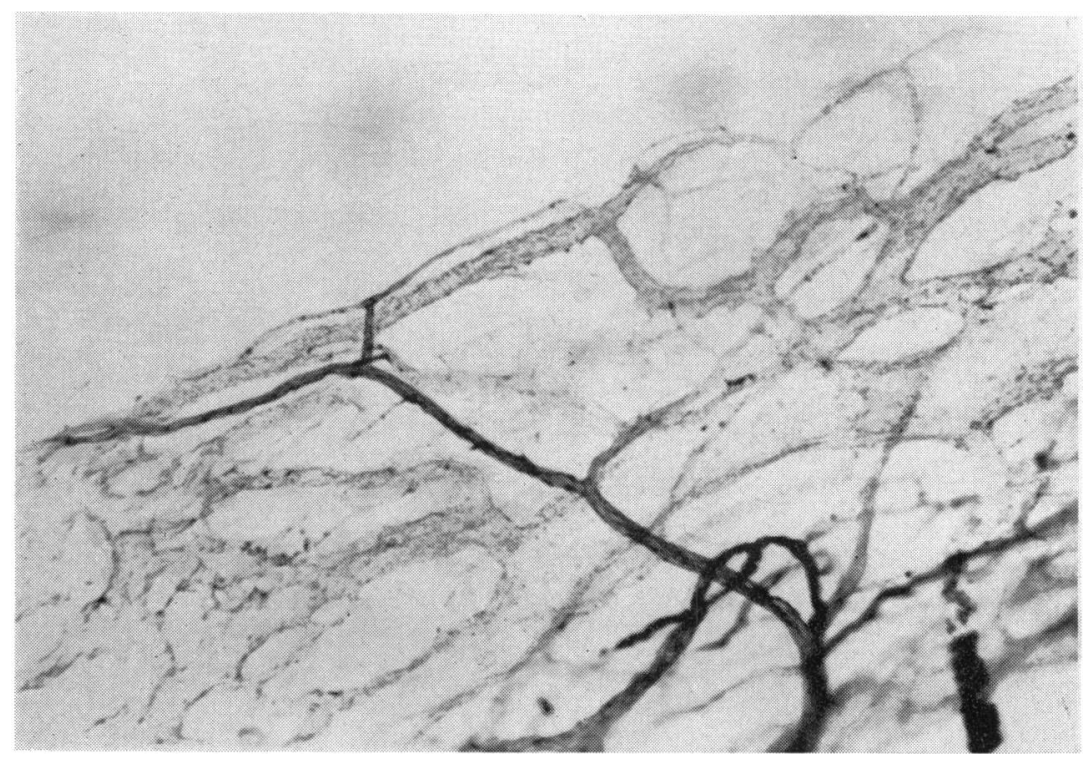

Fig. 6.-Staining of arterial system with Viacutan up to the level of the precapillary arterioles. Capillaries and venous system are coated with Monolitered particles. $\times 100$.

In the freshly killed rabbit, Viacutan causes marked arteriolar spasm (Fig. 7), unless preceded by formalin.

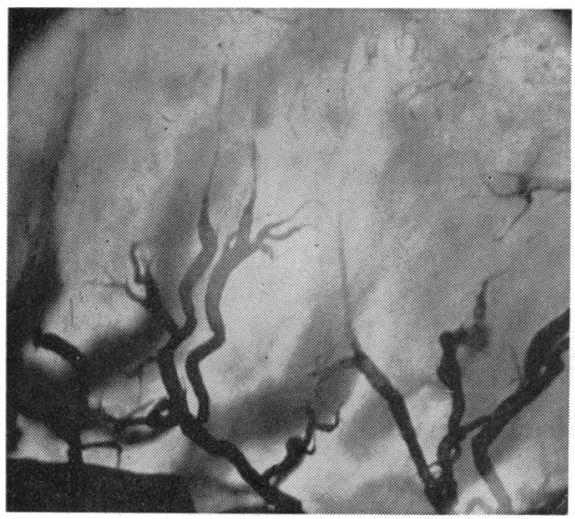

Fig. 7.-Spasm caused by Viacutan in iris arterioles not previously perfused with formalin. $\times 25$.

Conversely, if preceded by too much formalin, it stains some of the capillary and venous network. This overlapping of the capillary and venous system is not misleading as the stain in these vessels is light and can easily be traced back to the more darkly stained parent arteriole. 
The main arterial trunks are:

(1) The intra-ocular long ciliary arteries (Fig. 8), medial and lateral.

(2) The extra-ocular arteries coming from the recti muscles.

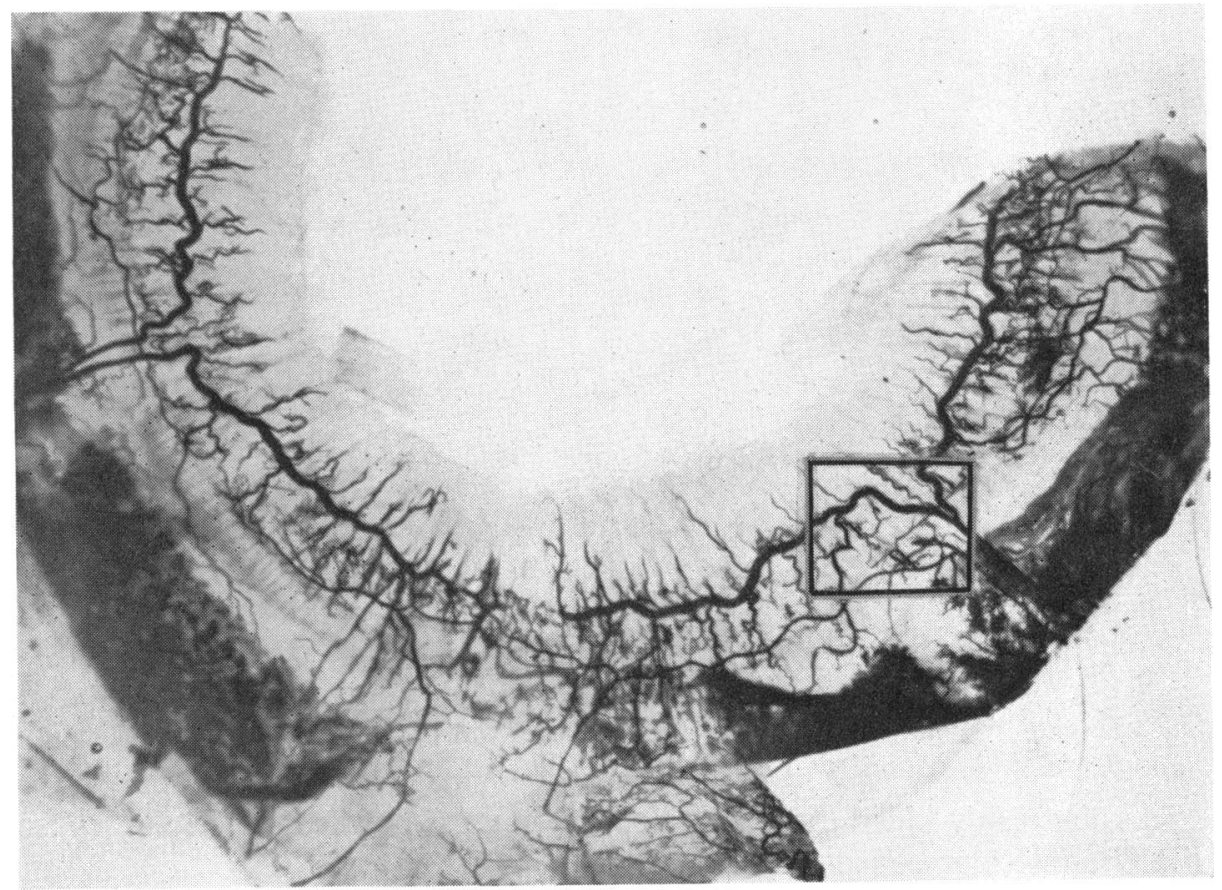

FIG. 8.-Arterial system stained with Viacutan. $\times 5$.

Each long ciliary artery, unpaired by a vein, enters the globe posteriorly, crosses anteriorly through the choroid, bifurcates into two branches and continues forward in the choroid to end at the arterial circle of the iris (Fig. 9).

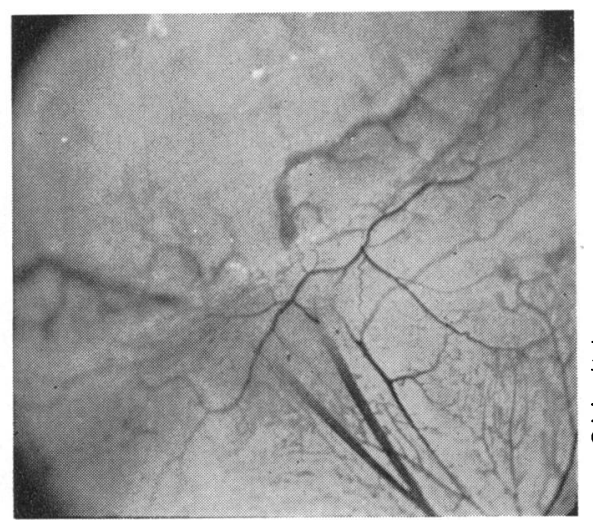

FIG. 9.-Eye perfused with Monolite red and Viacutan after exposure to ultraviolet light. Note perforating limbal vessel from branch of long ciliary artery. $\times 5$. 
From the arterial circle, short branches go proximally to supply the ciliary processes. Also from the arterial circle, narrower branches, both long and short, supply the iris tissue. Usually a small tributary arises from the long ciliary artery, or one of its choroidal branches, and courses anteriorly to perforate the sclera at the limbus and so supply the superficial limbal plexus (Figs 9 and 10).

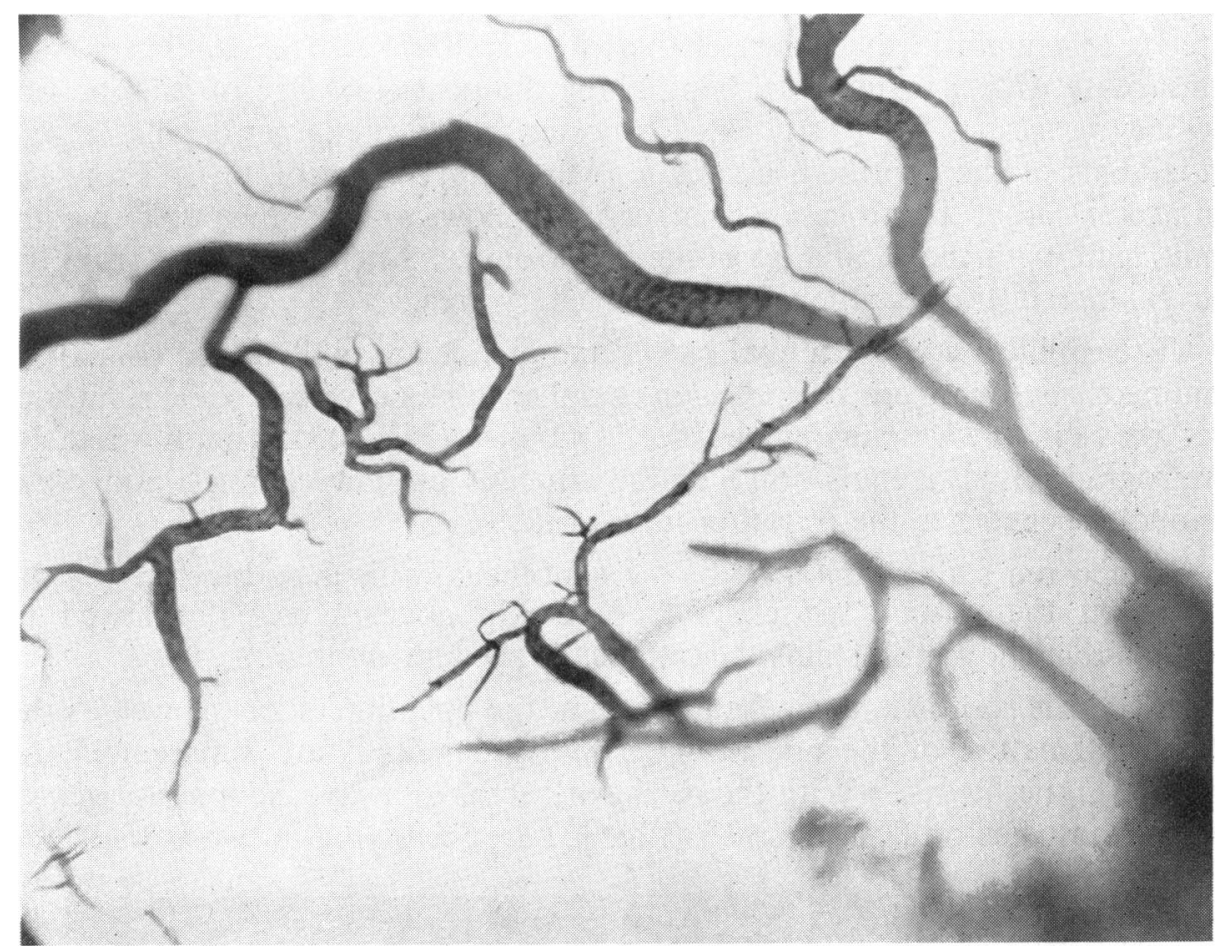

FIG. 10.-Higher power of rectangular area (Fig. 8), showing arterial branch, lower centre, of a long ciliary artery piercing the sclera at the limbus. $\times 40$.

This perforating artery is usually separated from extra-ocular branches which have come from the recti muscles by a capillary plexus. If, however, direct anastomoses exist between these two arterial systems, the perforating artery takes a spiral or kinked course (Fig. 11) as it passes through the scleral wall.

Fig. 11.-Spiral scleral course of perforating branch of long ciliary artery anastomosing with extra-ocular artery from rectus muscle. $\times 10$.

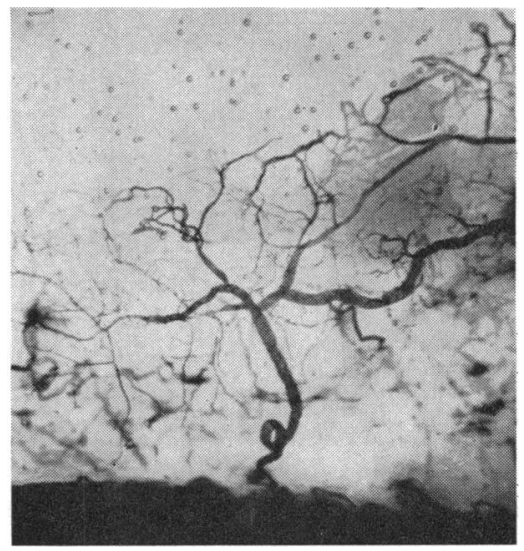


The extra-ocular arteries from the recti muscles spread out along the limbus and occasionally send a perforating branch to communicate with the arterial circle of the iris.

The walls of the capillaries and veins are coated by a thin film of Monolite red (Figs 3,6). The lumen of these vessels, however, is for the most part clear (Fig. 6). If Monolite red is present in the arterial system, it is masked by the more dominant Viacutan. In the ciliary processes, Monolite red tends to remain in the lumen of the wide capillaries. The capillaries communicate with a main vessel lying along the posterior edge of each fin-like ciliary process. This vessel extends posteriorly to the larger capillaries and the veins of the choroidal circulation (Fig. 12a, $b$, see col. pl.). From the anterior end of the process a small capillary flows towards the pupil, to communicate with the capillaries of the iris stroma. Attenuated veins from the uveal system traverse the sclera in the ciliary area.

The capillaries of the limbal plexus and arcade are pink, or red (Fig. 3), if unmixed by dye from the anterior chamber. These vessels join tributaries of the veins accompanying the recti muscles (Fig. 4). Monolite red, by retrograde flow, colours some of the aqueous outflow channels and occasionally penetrates the filtration meshwork.

Congo red stains the nuclei of the endothelial cells deep blue, giving the walls of the outflow vessels a leopard-skin appearance. If allowed to continue, the dye also stains the cell membrane and cytoplasm.

If the iris root is left attached to sclera, the open interstices as well as the syncytial nature of the blue staining individual fibres are apparent in the filtration meshwork. With the meshwork scraped away, the inner openings of the aqueous outflow channels (Fig. 13) are seen lying in two rows which

(a)

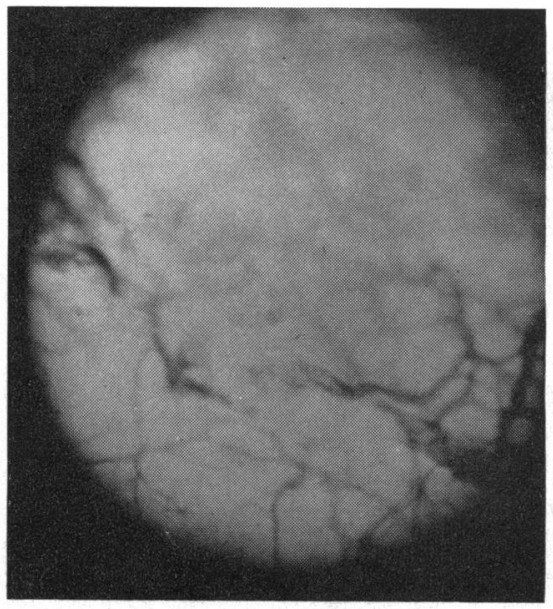

FIG. $13(a$ and $b$ ).-Stereophotomicrograph, showing inner openings of aqueous outflow channels after filtration meshwork has been scraped away. $\times 35$. 
encircle the inner aspect of the globe, one deep to the corneo-scleral sinus and one slightly more posterior. There are approximately 130 such openings.

In the rabbit's eye there is no such rich intrascleral network of aqueous outflow or arterial channels as is found just external to the canal of Schlemm in man. Instead, the channels take a more direct course to the surface (Figs 1 and 2), the anterior group leading to the superficial limbal plexus, and the posterior group leading to occasional intrascleral, but mostly extraocular veins. Approximately half of these vessels communicate with each other in both their intrascleral and their extrascleral course. No Congo red enters the choroidal circulation. As in the human eye, no direct communication is found between the aqueous outflow vessels and the arterial system.

The surface aqueous outflow vessels and most of the vessels of the limbal plexus are coloured blue or purple (Fig. 3), depending on the route and volume of fluid traffic. Nearly all of the surface aqueous vessels join the veins of the recti muscles (Fig. 4).

\section{Discussion}

Monolite red, Viacutan, and Congo red are all compatible in water, their common vehicle. The first coats the vessel walls and the other two stain the endothelium. This technique does not depend on a cast remaining in the vessel and consequently one colour cannot unintentionally be displaced by another. While the exact vascular fluid traffic of the living animal under various vasomotor controls cannot be duplicated in vitro, the potential availability of vascular communications is still demonstrated. The sites of overlapping of blue and red colours are likely sites of variability in fluid traffic in the living eye.

The major problems of water leaking into the tissues and of Viacutan diffusing into the anterior chamber blocking the filtration meshwork are largely prevented by the presence of gelatin in some of the perfusates. This, combined with judicious control of fluid volumes and pressures, produces good results. The technique has been adapted to other species by altering the site of injection, and the perfusion volume and pressure.

Viacutan is a silver-containing molecule, silver dinaphthylmethane disulphonate. Monolite red is a monoazo pigment referred to as Colour Index Pigment Red, 7. The colloidal particles are for the most part between 0.1 and $1.0 \mathrm{~m} \mu$. in diameter. During perfusion the particles precipitate as they contact the vessel walls, lining the endothelium with a red coat. Dispersol OG, in the proportions indicated in the method, protects Monolite red sufficiently to prevent it from coating laboratory glassware but not from coating the endothelium of the vessel walls.

Congo red is an indicator dye which undergoes a reversible reaction forming a blue precipitate at $\mathrm{pH} 4$. It is comprised of a chain of six benzene rings and, because of its molecular configuration, it passes the filtration meshwork 
with comparative ease; yet it diffuses through the capillary walls slowly enough to be precipitated by acid at the endothelial lining.

\section{Summary}

(1) A technique is described in which the arterial, venous, and aqueous outflow systems are stained each with a contrasting colour by a relatively simple method requiring less than one hour to complete from the time of initial perfusion.

(2) The arterial system is stained golden brown as far distally as the precapillary arteriole by a silver-containing compound, Viacutan, injected arterially.

(3) The endothelial lining of the capillary and venous system is coated red by Monolite red 4RHVS particles, also injected arterially.

(4) The aqueous outflow channels are stained blue by the injection of Congo red into the anterior chamber which perfuses through the filtration meshwork. If this technique is applied to other species obviously no cannulization of a canal of Schlemm is required.

(5) Where aqueous and venous flow merge, a fourth colour, purple, is produced and layering or stratification is occasionally evident.

(6) The specimen is cleared and viewed stereoscopically, allowing a threedimensional appreciation of the multiple vascular relationships.

The authors wish to express their thanks to Dr. Francis Burger of Telegraph Condenser Company Canada Limited, and to Imperial Chemical Industries Limited, Manchester, England, for their interest and advice in the development of this technique, and to Mrs. Lisa Schubert for technical assistance.

\section{REFERENCES}

AsCher, K. W. (1942). Amer. J. Ophthal., 25, 1174.

Ashton, N. (1951). Brit. J. Ophthal., 35, 291. (1952). Ibid., 36, 265. and SMith, R. (1953). Ibid., 37, 577.

Cibis, P. A., and Yamashita, T. (1959). Amer. J. Ophthal., 48, No 5, pt 2, 465. CoHAN, B. E. (1958). A.M.A. Arch. Ophthal., 60, 110.

- (1960). Ibid., 63, 489.

Goldmann, H. (1946). Ophthalmologica (Basel), 111, 146.

Greaves, D. P., and Perkins, E. S. (1952). Brit. J. Ophthal., 36, 258.

HAUSLER, H. R., and Sibay, T. M. (1960). Ibid., 44, 46.

LeBER, T. (1873). v. Graefes Arch. Ophthal., 19, pt 2, p. 87.

Schwalbe, G. (1870). Arch. mikrosk. Anat., 6, 262. 